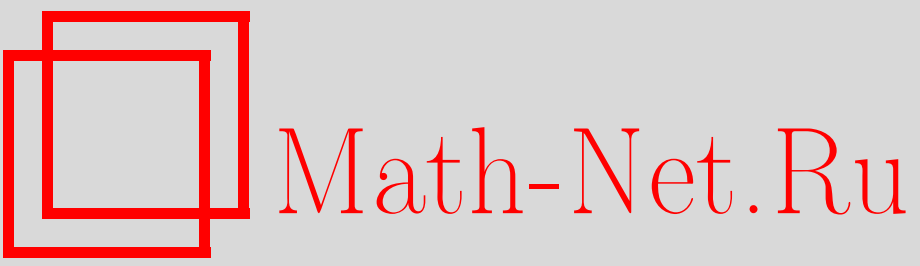

Нгуен Ван Хьеу, Квазипотенциальный подход в квантовой хромодинамике, TMФ, 2002, том 132, номер 2, 295-299

DOI: https://doi.org/10.4213/tmf362

Использование Общероссийского математического портала Math-Net.Ru подразумевает, что вы прочитали и согласны с пользовательским соглашением

http://www . mathnet.ru/rus/agreement

Параметры загрузки:

IP : 107.22 .136 .117

26 апреля 2023 г., 04:55:28 
ТЕОРЕТИЧЕСКАЯ

И МАТЕМАТИЧЕСКАЯ

ФИЗИКА

Том 132, № 2

август, 2002

(C) 2002 г.

Нгуен Ван Хьеу*

\section{КВАЗИПОТЕНЦИАЛЬНЫЙ ПОДХОД В КВАНТОВОЙ ХРОМОДИНАМИКЕ}

Квазипотенциальный метод Логунова-Тавхелидзе применяется к изучению некоторых процессов квантовой хромодинамики при конечных плотности и температуре. Выводится общее динамическое уравнение цветной сверхпроводимости. Изучается спонтанное нарушение киральной, ароматной и цветной симметрий. Обсуждается возможность построения полевой теории составных трехкварковых систем.

Ключевые слова: квантовая хромодинамика; киральная, ароматная и цветная симметрии; трехкварковые системы.

Около сорока лет назад Логунов и Тавхелидзе предложили использовать в квантовой теории поля квазипотенциальньй подход [1]-[4] - универсальный метод исследования связанных состояний релятивистских двухчастичных систем и асимптотического поведения амплитуд рассеяния при высоких энергиях, основанный на введении некоторого зависяшего от энергии комплексного квазипотенциала и использовании релятивистского уравнения шредингеровского типа с квазипотенциалом. Автору этой статьи выпало счастье работать в то время в возглавляемом А. А. Логуновым научном коллективе в Объединенном институте ядерных исследований. Накопленные тогда знания и опыт научной работы оказались весьма ценными. В данной работе предлагается применить квазипотенциальный метод Логунова-Тавхелидзе к исследованию двух актуальных проблем квантовой хромодинамики (КХД) для материи с конечными плотностью и температурой - цветной сверхпроводимости и спонтанного нарушения симметрии. Обсуждается также возможность построения полевой теории составных трехкварковых систем.

\section{1. ЦВЕТНАЯ СВЕРХПРОВОДИМОСТЬ}

Сверхпроводящее спаривание кварков в КХД может происходить благодаря двум механизмам: глюонному обмену и индуцированным инстантонами кварк-кварковым взаимодействиям. Вклад каждого из этих механизмов в отдельности в цветную сверхпроводимость рассматривался во многих работах [5]-[15]. В обшем случае необходимо учитывать оба указанных механизма одновременно. Для этой цели пригоден квазипотенциальный метод с использованием функциональных интегралов.

${ }^{*}$ Институт физики, Национальный центр естественных наук и технологий, Факультет технологии, Ханойский национальный университет, Ханой, Вьетнам 
Мы будем использовать формализм с мнимым временем. Кратко запишем:

$$
x=(\mathbf{x}, \tau), \quad \int d x=\int_{0}^{\beta} d \tau \int d \mathbf{x}, \quad \beta=\frac{1}{k T},
$$

где $k$ - постоянная Больцмана и $T$ - абсолютная температура. Обозначим через $\psi_{A}$, $\bar{\psi}^{A}$ кварковое поле и сопряженное ему, где индекс $A=(\alpha, a, i)$ описывает совокупность спинорного индекса $\alpha=1,2,3,4$, индекса цветной симметрии $a=1,2, \ldots, N_{\mathrm{c}}$ и индекса ароматной симметрии $i=1,2, \ldots, N_{\mathrm{f}}$. Исходным пунктом для построения теории цветной сверхпроводимости является статсумма для системы взаимодействующих кварков

$$
\begin{aligned}
& Z=\int[D \psi][D \bar{\psi}] \exp \left\{-\int d x \bar{\psi}^{A}(x) L_{A}^{B} \psi_{B}(x)\right\} \times \\
& \times \exp \left\{\frac{1}{2} \int d x \int d y \bar{\psi}^{A}(x) \bar{\psi}^{C}(y) V_{C A}^{B D}(x-y) \psi_{D}(y) \psi_{B}(x)\right\},
\end{aligned}
$$

где

$$
L_{A}^{B}=\delta_{a}^{b} \delta_{i}^{j}\left[\gamma_{4}\left(\frac{\partial}{\partial x}-\mu\right)+\gamma \nabla+M\right]_{\alpha}^{\beta},
$$

$\mu$ и $M$ - химический потенциал и масса голого кварка, соответственно, а $V_{C A}^{B D}(x-y)-$ квазипотенциал. Ради простоты рассмотрим приближение, в котором квазипотенциал зависит только от разности двух координат $(x-y)$. Наши рассуждения легко применить к общему случаю, когда комплексный квазипотенциал зависит от разностей различных координат всех четырех кварковых полей.

Введем билокальные биспинорные составные поля $\Phi_{A C}(x, y)$ и сопряженные им $\bar{\Phi}^{A C}(x, y)$, подчиняющиеся статистике Ферми-Дирака:

$$
\Phi_{C A}(y, x)=-\Phi_{A C}(x, y), \quad \bar{\Phi}^{C A}(y, x)=-\bar{\Phi}^{A C}(x, y),
$$

и применим преобразование Хаббарда-Стратоновича. Тогда функцию распределения $Z$ можно представить в виде функционального интеграла по составным полям

$$
Z=\int[D \Phi][D \bar{\Phi}] \exp \left\{S_{\text {eff }}[\Phi, \bar{\Phi}]\right\}
$$

с некоторым эффективным действием $S_{\text {eff }}[\Phi, \bar{\Phi}]$. Уравнения для составных полей, вытекающие из принципа экстремального действия

$$
\frac{\delta S_{\text {eff }}[\Phi, \Phi]}{\delta \bar{\Phi}^{A C}(x, y)}=0
$$

представляют собой обобшенные уравнения БКШ для параметров порядка цветной сверхпроводимости. В частных случаях отдельных механизмов кварк-кваркового спаривания они были рассмотрены в работах [5]-[14]. Квазипотенциальный подход с использованием функционнальных интегралов [15] представляет собой уникальный метод для изучения общего случая, когда оба механизма кварк-кваркового спаривания действуют одновременно. 


\section{2. СПОНТАННОЕ НАРУШЕНИЕ СИММЕТРИИ}

Наличие ненулевых параметров порядка $\Phi_{A B}$ приводит к спонтанному нарушению соответствуюших свойств симметрий системы взаимодействуюших кварков с конечными плотностью и температурой. Поскольку эти параметры порядка являются спинорами второго ранга (с двумя индексами $a, b=1,2,3$ ) группы цветной симметрии $S U(3)_{\mathrm{c}}$, они вносят вклад в матричные элементы различных процессов, нарушаюших цветную симметрию, в том числе процессов преврашения фотона в глюон и обратно (фотон-глюонное смешивание) [16]. Кроме того, нарушение калибровочной цветной сим-

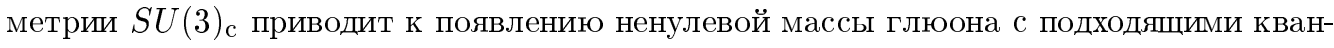
товыми числами группы $S U(3)_{c}$. Из-за фотон-глюонного смешивания этот массивньй глюон квантовой хромодинамики для материи с конечньми плотностью и температурой распадается на электрон-позитронные пары. Данный процесс может служить экспериментальным признаком образования кварк-глюонной плазмы.

Квантовая хромодинамика с нулевой массой голых кварков обладает киральной симметрией. Наличие ненулевых параметров порядка $\Phi_{A B}$ приводит к появлению ненулевой массы кварков и тем самым спонтанно нарушает киральную симметрию. Другим механизмом спонтанного нарушения киральной симметрии является кварк-антикварковое спаривание.

Для изучения кварк-антикваркового спаривания [17], [18] воспользуемся функцией распределения (1) в несколько ином виде:

$$
\begin{aligned}
Z= & \int[D \psi][D \bar{\psi}] \exp \left\{-\int d x \bar{\psi}^{A}(x) L_{A}^{B} \psi_{B}(x)\right\} \times \\
& \times \exp \left\{\frac{1}{2} \int d x \int d y \bar{\psi}^{A}(x) \psi_{B}(y) \mathcal{U}_{A C}^{B D}(x-y) \bar{\psi}^{C}(y) \psi_{D}(x)\right\},
\end{aligned}
$$

где квазипотенциал обозначен как $\mathcal{U}_{A C}^{B D}(x-y)$. Введем составные билокальные мезонные поля $\Phi_{B}^{A}(x, y)$ с трансформационными свойствами, которыми обладают произведения $\bar{\psi}^{A}(x) \psi_{B}(y)$. Посредством преобразования Хаббарда-Стратоновича представим функцию распределения (5) в виде функционального интеграла по мезонными полям

$$
Z=\int[D \Phi] \exp \left\{S_{\mathrm{eff}}[\Phi]\right\}
$$

с некоторым эффективным действием $S_{\text {eff }}[\Phi]$. Уравнения для составных мезонных полей, вытекающие из принципа экстремального действия

$$
\frac{\delta S_{\mathrm{eff}}[\Phi]}{\delta \Phi_{B}^{A}(x, y)}=0,
$$

являются динамическими уравнениями для определения параметров порядка системы взаимодействующих кварков при наличии кварк-антикваркового спаривания. Если эти параметры порядка являются синглетами групा цветной и ароматной симметрий, то 
они приводят только к спонтанному нарушению киральной симметрии. Было показано, что в случае группы ароматной симметрии $S U(2)$ сушествуют ненулевые параметры порядка, образующие триплет этой группы и, следовательно, ароматная симметрия спонтанно нарушается [18]. Имеется, однако, вырождение между фазами с триплетным и синглетным параметрами порядка, если кварк-кварковые взаимодействия индуцированы инстантонами.

\section{3. ОБРАЗОВАНИЕ ТРИКВАРКА}

Квазипотенциальный поход оказывается весьма удобным методом для изучения образования трикварка - связанного состояния системы трех кварков в КХД. Для этой цели воспользуемся функциональным интегралом системы взаимодействующих кварков в виде

$$
\begin{aligned}
Z= & \int[D \psi][D \bar{\psi}] \exp \left\{-i \int d^{4} x \bar{\psi}^{A}(x)\left[(\hat{\gamma})_{A}^{B}+m \delta_{A}^{B}\right] \psi_{B}(x)\right\} \times \\
& \times \exp \left\{\frac{i}{6} \int d^{4} x \int d^{4} y \int d^{4} z \int d^{4} u \int d^{4} v \int d^{4} w \bar{\psi}^{F}(w) \bar{\psi}^{E}(v) \bar{\psi}^{D}(u) \times\right. \\
& \left.\times V_{D E F}^{C A B}(u, v, w ; z, y, x) \psi_{A}(x) \psi_{B}(y) \psi_{C}(z)\right\}
\end{aligned}
$$

с некоторым квазипотенциалом взаимодействия между тремя кварками $V_{D E F}^{C B}(u, v, w$; $z, y, x)$. Введя составные трилокальные триспинорные барионные поля $\Psi_{A B C}(x, y, z)$ и сопряженные им $\bar{\Psi}_{A B C}(x, y, z)$ и осуществив соответствуюшее преобразование Хаббарда-Стратоновича, получим функциональный интеграл (8) в виде

$$
Z=\int[D \Psi][D \bar{\Psi}] \exp \left\{i S_{\mathrm{eff}}[\Psi, \bar{\Psi}]\right\}
$$

с некоторым эффективным действием $S_{\text {eff }}[\Psi, \bar{\Psi}]$. Уравнения для составных полей, вытекающие из принципа экстремального действия

$$
\frac{\delta S_{\text {eff }}[\Psi, \bar{\Psi}]}{\delta \bar{\Psi}^{A B C}(x, y, z)}=0
$$

составляют систему динамических интегральных уравнений для связанных состояний системы трех кварков. Они были получены в работе [19]. Дальнейшее изучение их решений представляет собой интересную проблему физики адронов. 


\section{Список литературы}

[1] A. A. Logunov, A. N. Tavkhelidze. Nuovo Cimento. 1963. V. 29. P. 380.

[2] A. A. Logunov, A. N. Tavkhelidze, I. T. Todorov, O. A. Khrustalev. Nuovo Cimento. 1963. V. 30. P. 134.

[3] A. A. Logunov, Nguyen Van Hieu, O. A. Khrustalev. Nucl. Phys. 1964. V. 50. P. 295.

[4] Nguyen Van Hieu, R. N. Faustov. Nucl. Phys. 1964. V. 53. P. 337.

[5] M. Alford, K. Rajagopal, F. Wilczek. Phys. Lett. B. 1998. V. 422. P. 247; Nucl. Phys. B. 1999. V. 537. P. 443.

[6] T. Schäfer, F. Wilczek. Phys. Rev. Lett. 1999. V. 82. P. 3956; Phys. Lett. B. 1999. V. 450. P. 325.

[7] R. Rapp, T. Schäfer, E. Shuryak, M. Velkovsky. Phys. Rev. Lett. 1998. V. 81. P. 53.

[8] N. Evans, S. Hsu, M. Schwetz. Nucl. Phys. B. 1999. V. 551. P. 275; Phys. Lett. B. 1999. V. 449. P. 281.

[9] D. T. Son. Phys. Rev. D. 1998. V. 59. P. 094019.

[10] G. W. Carter, D. Diakonov. Phys. Rev. D. 1999. V. 60. P. 01004.

[11] A. Chodos, H. Minakata, F. Cooper. Phys. Lett. B. 1999. V. 449. P. 260.

[12] T. Schäfer, F. Wilczek. Phys. Rev. D. 1999. V. 60. P. 074014; 114033.

[13] V. A. Miransky, I. A. Shovkovy, L. C. Wijewardhava. Phys. Lett. B. 1999. V. 468. P. 270.

[14] R. Casalbuoni, R. Gatto. Phys. Lett. B. 1999. V. 464. P. 111.

[15] Nguyen Van Hieu. Basics of Functional Integral Technique in Quantum Field Theory of Many-Body Systems. Hanoi: VNUH Pub., 1999.

[16] Nguyen Van Hieu, Pham Xuan Yem. Phys. Rev. D. 2001. V. 64. P. 074009.

[17] M. Harada, A. Shibata. Phys. Rev. D. 1998. V. 59. P. 014010.

[18] Nguyen Van Hieu, Nguyen Hung Son, Ngo Van Thanh, Hoang Ba Thang. Adv. Nat. Sci. 2000. V. 1. P. 61; hep-ph/0001251.

[19] Nguyen Van Hieu. Adv. Nat. Sci. 2001. V. 2. P. 115. 